\title{
Liquid Crystal Orientation Transition on Microtextured Substrates
}

\author{
Baoshe Zhang, Fuk Kay Lee, Ophelia K. C. Tsui, ${ }^{*}$ and Ping Sheng \\ Department of Physics and Institute of Nano Science and Technology, Hong Kong University of Science and Technology, \\ Clear Water Bay, Kowloon, Hong Kong
}

(Received 14 June 2003; published 18 November 2003)

\begin{abstract}
A uniform alignment of liquid crystal (LC) with finite pretilt was observed on microtextured substrates that were lithographically fabricated with alternating horizontal and vertical corrugations. As the period of alternation was decreased toward $0.8 \mu \mathrm{m}$, the nematic LC alignment on these substrates changed from inhomogeneous in plane, copying the substrate corrugations, to a uniform configuration with a large pretilt of $\sim 40^{\circ}$. This transition is pertinent to a frustrated boundary wherein a lowering in the LC elastic energy due to spatial variation in the LC orientation compromises an increase in the surface anchoring energy. A model based on this idea demonstrates good agreement with the experiment. This result may open up a new arena for tailoring substrate characteristics for LC alignment.
\end{abstract}

DOI: 10.1103/PhysRevLett.91.215501

PACS numbers: 61.30.Hn, 61.30.Dk, 61.30.Eb

Substrate conditioning for the alignment of liquid crystal (LC) molecules is essential to the operation of LC displays. Most existing techniques used to produce the alignment effect involve uniform treatment of the substrate surface. An unconventional approach to uniform LC alignment, based on inhomogeneous patterning of the substrate, was proposed some time ago [1,2]. The idea is to create a situation wherein the LC director, in coping with the inhomogeneous substrate pattern, acquires so large an elastic energy that the LC has to compromise with a uniform configuration. In general, this frustrated boundary condition can be achieved by making the pattern period small enough. A few versions of this idea, including the use of inhomogeneous surfactant treatment [1,3] and inhomogeneous rubbing [4], have been reported successful. More recently, an interesting application of these substrates in multistable display, which may potentially lead to lower energy consumption, was demonstrated $[5,6]$. In these demonstrations, the LC director makes zero pretilt with the substrate, and the switching between different stable LC states was achieved by varying an in-plane electric field. However, out-of-plane switching is preferred since the electric field can be more uniform. But that requires the LC pretilt to be finite [7], which has not been found in any reported embodiments $[1,3,4]$. In yet some designs, a large LC pretilt is crucial as it widens the range of LC elastic constants usable, which would otherwise be too narrow to be practical [8]. Here we show that on lithographically made substrates with alternating horizontal and vertical corrugations, the nematic alignment undergoes an orientational transition that results in a large pretilt angle $\left(\sim 40^{\circ}\right)$. A simple model used to simulate the transition not only showed good agreement with the experiment but also revealed that the polar surface energies of the frustrated boundary are only $\sim 1 / 10$ of typical values. Weak surface anchoring energies are virtuous for lowering device energy consumption and response time [9].
Figure 1 shows a subset of the microtextured substrates employed in this study. They consist of arrays of parallel corrugations $85 \mathrm{~nm}(\mathrm{w}) \times 30 \mathrm{~nm}(\mathrm{~h})$ arranged alternatively along the $x$ and $y$ directions, having the period of alternation, $\lambda$, varied from 12 to $0.4 \mu \mathrm{m}$. The microtextured patterns, $\sim 23 \times 25 \mu \mathrm{m}^{2}$ in coverage, were fabricated by using the atomic force microscope (AFM) local oxidation technique [10] on $\mathrm{Si}(100)$ wafers, followed by selective etching in aqueous potassium hydroxide solution. The substrates were then made into LC cells using $\left(\mathrm{In}_{2} \mathrm{O}_{3}\right)_{9} \mathrm{SnO}_{2}$ (ITO)/glass slides coated with unidirectionally rubbed polyimide (PI) for the upper cell walls. The LC, $4^{\prime}$-n-pentyl-4-cyanobiphenyl (5CB), was filled into the $5-\mu \mathrm{m}$ cell gap by capillarity.

Figure 2 shows reflection images of the cells between crossed polarizers with the entrance polarizer aligned parallel to the PI rubbing direction $(y)$. As seen, images of the two cells with the largest periods exhibit checkerboard patterns that duplicate the one on the substrate. In the layout of Fig. 2, the PI rubbing direction is along $y$ and
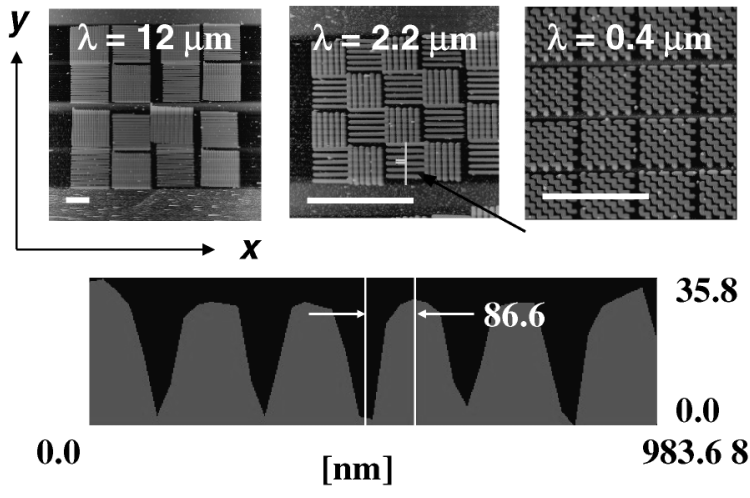

FIG. 1. AFM topographical images of several microtextured substrates we fabricated. The scale bars are $3 \mu \mathrm{m}$. The arrow in the $\lambda=2.2 \mu \mathrm{m}$ sample indicates from where the crosssectional profile in the lower panel was drawn. 


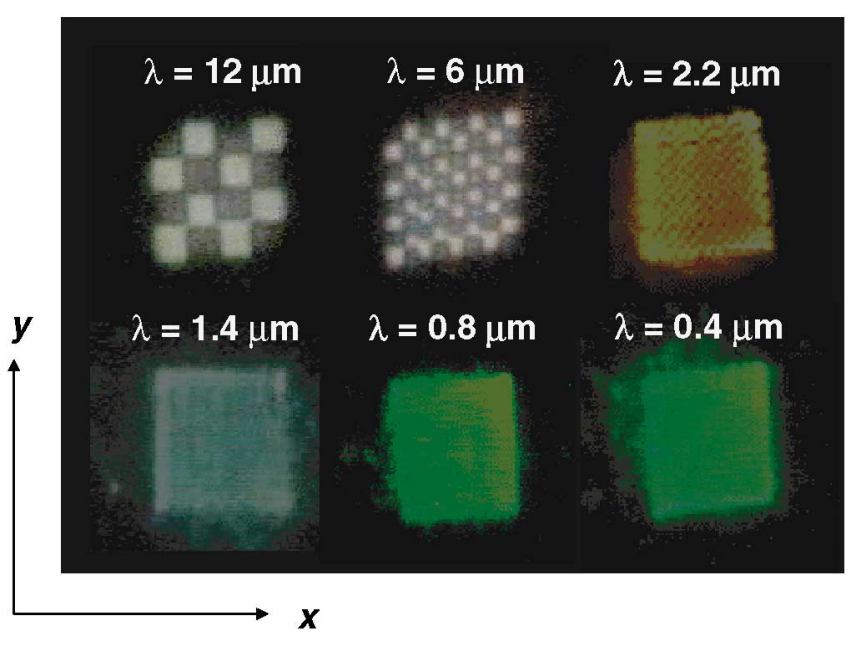

FIG. 2 (color). Optical reflection micrographs taken with crossed polarizers of LC cells made from a series of microtextured substrates. The upper plates of the cells are ITO/glass coated with PI uniaxially rubbed along $y$. The microtextures in this layout have the same orientation as those in Fig. 1.

the microtextured substrates have the same orientation as in Fig. 1. Optical measurements on a cell made of microtextured substrates with uniform parallel corrugations confirmed that the grooves impose an alignment of the LC director along the groove direction, consistent with Berreman's model [11] for minimum LC elastic energy. Accordingly, dark regions in the reflection images of Fig. 2 are due to uniform alignment of the LC along $y$, whereas the bright regions are due to a $90^{\circ}$ twist in the LC director traversing across the cell. As $\lambda$ is reduced, the checkerboard pattern becomes progressively more blurred and reveals different colors with different $\lambda$. Upon reaching $\lambda=0.8 \mu \mathrm{m}$, the cell stabilizes to a uniform greenish appearance. This observation strongly suggests a transition from an inhomogeneous state steadily to an optically uniform state that occurs near $\lambda=0.8 \mu \mathrm{m}$. However, $\lambda=0.8 \mu \mathrm{m}$ also coincides with where the checkerboards (whose size $\equiv \lambda / 2$ ) become unresolved in the present optical setup. To elucidate the true picture of the observed transition, we investigate the LC configuration for the optically uniform cells with $\lambda \leq 0.8 \mu \mathrm{m}$.

Inhomogeneity in the LC configuration imposed by a substrate modulation period $\lambda$ should decay vertically with a decay length of $\lambda / 4 \pi$ [11]. With $\lambda \leq 0.8 \mu \mathrm{m}$, this decay length is $\leq 0.06 \mu \mathrm{m}$ and hence $\ll$ the LC layer thickness, making the contribution of the inhomogeneous LC boundary layer to the overall appearance of the LC layer insignificant. We first determine the equilibrium azimuthal $\mathrm{LC}$ alignment in the relaxed region above the boundary layer. To do so, we examine the reflection images of the microtexture, with a layer of LC deposited on top, taken between crossed polarizers at different rotation angles $\psi$ (see the inset of Fig. 3). Since the upper interface is air, the azimuthal director orientation at the substrate $(\phi)$ is not changed through the LC layer.

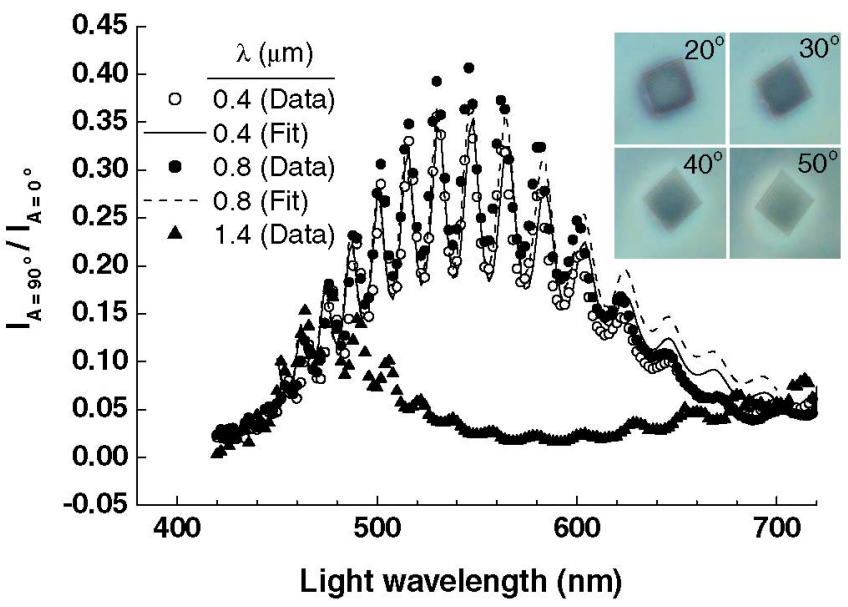

FIG. 3 (color). Inset: reflection images of a LC film freshly deposited on the $\lambda=0.8 \mu \mathrm{m}$ microtexture rotated at different angles as indicated, sandwiched between crossed polarizers. Main panel: measured (symbols) and simulated (continuous lines) $\mathrm{I}_{\text {crossed }} / \mathrm{I}_{\text {parallel }}$ of the $\lambda=0.4,0.8$, and $1.4 \mu \mathrm{m}$ LC cells.

The azimuthal alignment can be determined directly from the rotation angle that brings about the optical extinction of its own image. From Fig. 3, $\phi$ is about $30^{\circ}$. A detailed measurement of the reflected light intensity versus $\psi$ and curve fitting of the result to the expression $A \sin ^{2}(\psi-\phi)+B$ (where $A, B$ are constants) revealed that $\phi \sim 29^{\circ} \pm 3^{\circ}$. The same value of $\phi$ was similarly found for the $\lambda=0.8 \mu \mathrm{m}$ microtexture.

With the azimuthal orientation on the $\lambda \leq 0.8 \mu \mathrm{m}$ microtextures thus determined, we proceed to determine the pretilt angle, $\theta$. We measured the reflection spectra of the LC cells with the polarizer (aligned parallel with the PI rubbing direction) and the analyzer both crossed and parallel (denoted $\mathrm{I}_{\text {crossed }}$ and $\mathrm{I}_{\text {parallel }}$, respectively). The ratio of the two spectra, i.e., $\mathrm{I}_{\text {crossed }} / \mathrm{I}_{\text {parallel }}$, is shown in Fig. 3. Since optical spectra of the LC cells are results of their optical birefringence, which in turn arises from the director configuration, we model fit the data of Fig. 3 to deduce the LC alignment on the microtextures. First, the LC alignment at the upper boundary was assumed to be parallel with the PI rubbing direction, whereas that at the lower substrate was also assumed uniform but with an azimuthal angle fixed at $29^{\circ}$ and a pretilt angle $\theta$ as the only fitting parameter. With these boundary conditions, the LC configuration in the cell was determined through elastic energy minimization [12]. Then we used the experimentally determined ordinary and extraordinary refractive indices of $5 \mathrm{BC}(=1.532$ and 1.735 , respectively) to map out the dielectric tensor matrix across the cell, to which the $4 \times 4$ matrix formalism [14] was applied to compute the reflection spectra [15]. Results from curve fitting to the $\lambda=0.4$ and $0.8 \mu \mathrm{m}$ spectra indicate that the alignment in both cases have the same $\theta=40^{\circ} \pm 2^{\circ}$. This is in keeping with the fact that these two cells look alike in Fig. 2. On the other hand, the notably different spectrum from the $\lambda=1.4 \mu \mathrm{m}$ cell cannot be fitted 
with the above approach, suggesting its LC configuration to be optically nonuniform.

It is well known that obliquely evaporated $\mathrm{SiO}$ films can produce large LC pretilts (up to $25^{\circ}$ ) with size dependent on the topographic details of the film $[18,19]$ that generally contains $\mathrm{SiO}$ chains running perpendicular to the plane of incidence while on the chains there are perpendicular fine columns, slanted in the deposition direction. In films deposited at large angles $\left(>15^{\circ}\right)$ from the film surface, nematic LC was found to align parallel to the chains with zero pretilt $[18,19]$. But in films deposited at grazing incidence, features of the columns dominate those of the chains [18] whereupon the nematic LC director adopted an azimuthal alignment perpendicular to the chains with a finite pretilt [19]. In our structures produced by AFM local oxidation, the topography consists of simple upright grooves with no perpendicular columnar fine structure. Therefore, the LC director should lie parallel to the grooves with zero pretilt. This presumption has been confirmed by independent optical studies of LC anchoring on substrates fabricated by the AFM technique to contain purposefully only unidirectional grooves. It is thus not obvious how the LC configuration changes from one that is inhomogeneous, copying the local grooves with zero pretilt to one that is optically uniform with a large pretilt of $40^{\circ}$. Simple energy argument does not provide an obvious explanation.

To gain some insight into the transition, we have simulated the orientational transition through minimization of the total energies of the LC system [12], consisting of elastic energy, $U_{\mathrm{el}}$, and surface energy, $W$. For the elastic energy, we adopted the Frank-Oseen form [13],

$$
\begin{gathered}
U_{\mathrm{el}}=\int d^{3} r \frac{1}{2}\left\{k_{11}(\nabla \cdot \boldsymbol{n})^{2}+k_{22}(\boldsymbol{n} \cdot \nabla \times \boldsymbol{n})^{2}\right. \\
\left.+k_{33}(\boldsymbol{n} \times \nabla \times \boldsymbol{n})^{2}\right\},
\end{gathered}
$$

where $\boldsymbol{n}$ is the LC director, and $k_{11}=7 \times 10^{-12} \mathrm{~N}, k_{22}=$ $3.5 \times 10^{-12} \mathrm{~N}$, and $k_{33}=1 \times 10^{-11} \mathrm{~N}$ are, respectively, the splay, the twist, and the bend elastic constants [20]. For the surface energy, we used the commonly adopted form $[4,11,21]$,

$$
\begin{aligned}
W(\theta, \phi)= & \frac{1}{2} W_{\theta}^{(2)} \sin ^{2} \theta+\frac{1}{4} W_{\theta}^{(4)} \sin ^{4} \theta \\
& +\frac{1}{2} W_{\phi} \cos ^{2} \theta \sin ^{2} \phi(x, y),
\end{aligned}
$$

where $W_{\theta}^{(2)}$ and $W_{\theta}^{(4)}$ account for the polar anchoring strength [21] and $W_{\phi}$ for the azimuthal anchoring strength $[4,11]$ of the substrate on the LC. In Eq. (2), $\left(\theta_{0}=0^{\circ}, \phi_{0}=0^{\circ}\right)$ is assumed to be the easy director direction, i.e., the one that minimizes $W$. The maximum ranges of anchoring strengths found that can produce the observed transition were $W_{\theta}^{(2)} \leq 0.7 \times 10^{-6} \mathrm{~J} / \mathrm{m}^{2}$, $W_{\theta}^{(4)} \leq 1.3 \times 10^{-5} \mathrm{~J} / \mathrm{m}^{2}$, and $W_{\phi} \leq 1.1 \times 10^{-5} \mathrm{~J} / \mathrm{m}^{2}$. Figure 4 displays the transitions that resulted by putting $W_{\theta}^{(2)}=0, \quad W_{\theta}^{(4)}=1.3 \times 10^{-5} \mathrm{~J} / \mathrm{m}^{2}, \quad$ and $W_{\phi}=1.1 \times$ $10^{-5} \mathrm{~J} / \mathrm{m}^{2}$, which demonstrate excellent agreement with experiment. Our simulations can also reveal the LC configuration before and after the transition. We found that homogenization of the LC orientation commences even before the transition takes place so that the azimuthal angle of the LC at the substrate boundary has a rms value $\sqrt{\left\langle\left(\phi-\phi_{0}\right)^{2}\right\rangle} \cong 34^{\circ}$ and spread $\sqrt{\left\langle(\phi-\langle\phi\rangle)^{2}\right\rangle} \cong$ $17^{\circ}$ just before the transition (cf. $39^{\circ}$ and $6^{\circ}$, respectively, for these values after the transition). This can very well explain why images of the LC cells in Fig. 2 get increasingly blurred as the checkerboard period decreases. The transition is signified by a jump in the pretilt angle as Fig. 4 exemplifies. We found this jump to be attributable to two factors. One is a reduction in the elastic energy occurring when the in-plane LC director component is reduced. The other is a change of $W(\theta, \phi)$ from being monotonically increasing with $\theta$ to having a minimum near $\theta=$ $40^{\circ}$ as $\phi$ is increased towards $45^{\circ}$. The latter implies a set of relations governing the surface energy parameters, namely, $W_{\phi}=\gamma W_{\theta}^{(4)} / 2$ and $W_{\theta}^{(2)}=(0.5-0.8435 / \gamma) W_{\phi}$ with $\gamma \geq 1.687$. We have confirmed that energy parameters deduced from these relations always reproduce the $40^{\circ} \pm 5^{\circ} \theta$ jump at the transition. The magnitude of the parameters is determined by the position of the transition as well as by the amount of surface energy jump needed to balance the elastic energy release at the transition in a self-consistent manner. Larger surface energy suppresses homogenization of LC alignment, resulting in more tolerance to the building up of elastic energy and occurrence of the transition at smaller $\lambda$.

Although our model can give magnitudes of $W_{\phi}$ and $W_{\theta}^{(4)}$ that agree with theory [11] and published data $[21,22]$, the values of $W_{\theta}^{(2)}$ are at least $\sim 10$ times smaller than those reported in literature [21], leading to disagreement with the Rapini-Papoular (RP) potential [23], $W_{\mathrm{RP}} \sim\left(\boldsymbol{n} \times \boldsymbol{n}_{0}\right)^{2}$ [where $\boldsymbol{n}=(\theta, \phi)$ and $\boldsymbol{n}_{0}$ denotes the preferred direction and can be taken as $\left(0^{\circ}, 0^{\circ}\right)$ without loss of generality]. Measurements of polar energy are usually performed with $\phi=0^{\circ}$ whereby $W_{\mathrm{RP}}$ reduces

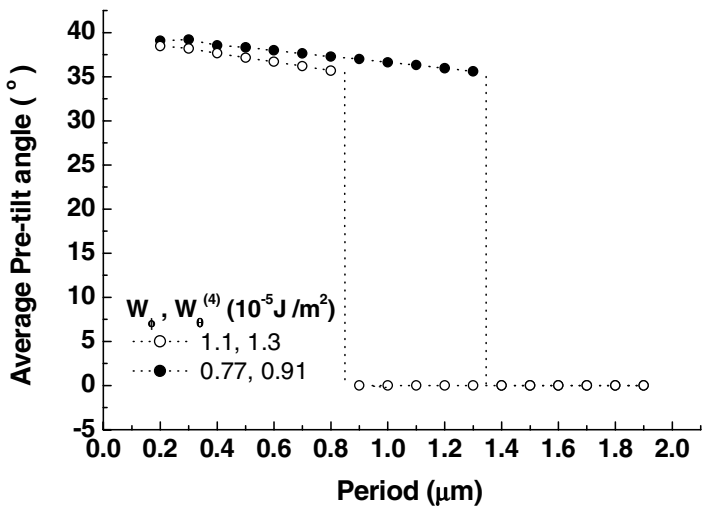

FIG. 4. Results from the free energy minimization calculations displayed as average pretilt angle vs $\lambda$, for two sets of $W_{\theta}$ and $W_{\phi}$ that give the two bounds to the observed transition. Dotted lines are guides to the eye. 
to $\sin ^{2} \theta$. But here $\phi$ is broadly distributed about $\langle\phi\rangle=$ $45^{\circ}$. Increasing $\phi$ from $0^{\circ}$ towards $90^{\circ}$ is noted to decrease the $\sin ^{2} \theta$ coefficient of $W_{\mathrm{RP}}$ steadily to 0 , which is unphysical. Thus the RP potential may need to be supplemented in cases where $\phi \neq 0^{\circ}$, perhaps by a reduction of the $\sin ^{2} \theta$ term and addition of a nontrivial $\sin ^{4} \theta$ term; both are found in our result.

Notably, the nature of LC anchoring in the present system is different from that of the more commonly encountered homogeneous surfaces on which the axis of symmetry is constant everywhere. In this model, the microtexture boundary has been treated as a twodimensional (2D) surface, but in reality it is 3D. Thence, the boundary conditions revealed by our model may correspond to a cross section in the actual 3D boundary. The LC director residing within the grooved layer is forced to deform according to the checkerboard pattern due to physical confinement by and chemophysical interactions with the topography walls, which in turn aligns the LC above through elastic interactions. Elastic interactions of this sort can often dominate the polar anchoring properties, giving rise to a dependence of the LC pretilt on details of the substrate topography $[18,19]$. Such a picture of the surface potential, with its origin lying in the elastic energy of a thin boundary layer adjacent to the substrate, gains support from the observed azimuthal anchoring at $29^{\circ}$ : Within this boundary layer, the in-plane variation of the director orientation relaxes by virtue of the restoring force $=-\nabla U_{\mathrm{el}}$, beyond which the LC adopts a uniform alignment. From Fig. 2, the LC cells are not exactly square but have an aspect ratio of 1:0.85 $(y: x)$. The in-plane restoring force experienced by the LC $\left(=\nabla U_{\mathrm{el}} \sim 1 / \lambda^{3}\right)$ will accordingly take on a ratio of $0.6: 1$ for its $y: x$ components. This would lead to an azimuthal anchoring at $31^{\circ}$, in good agreement with the experiment. On the other hand, LC deposited on patterns with $y: x$ close to 1 reveals an azimuth of $\sim 45^{\circ}$ as required.

We thank Professor H. S. Kwok and Professor W. Y. Tam for the use of their equipment. Useful discussions with Professor V. G. Chigrinov and Professor H. S. Kwok are acknowledged. This work is partially supported by RGC Grants No. HKUST6133/97P and No. HKUST6150/01P.

*Corresponding author.

Electronic address: phtsui@ust.hk

[1] H. L. Ong, A. J. Hurd, and R. B. Meyer, J. Appl. Phys. 57, 186 (1985).

[2] T. Z. Qian and P. Sheng, Phys. Rev. Lett. 77, 4564 (1996).

[3] B. Lee and N. A. Clark, Science 291, 2576 (2001).

[4] J. Kim, M. Yoneya, J. Yamamoto, and H. Yokoyama, Mol. Cryst. Liq. Cryst. 367, 151 (2001).

[5] J. Kim, M. Yoneya, J. Yamamoto, and H. Yokoyama, Appl. Phys. Lett. 78, 3055 (2001).

[6] J. Kim, M. Yoneya, and H. Yokoyama, Nature (London) 420, 159 (2002).
[7] See, for example, D.W. Berreman and W. R. Heffner, J. Appl. Phys. 52, 3032 (1981); R. Barberi, M. Giocondo, and G. Durand, Appl. Phys. Lett. 60, 1085 (1992).

[8] V. G. Chigrinov (private communication).

[9] G. P. Bryan-Brown, E. L. Wood, and I. C. Sage, Nature (London) 399, 338 (1999).

[10] F. K. Lee, G. H. Wen, X. X. Zhang, and O. K. C. Tsui, J. Vac. Sci. Technol. B 21, 162 (2003).

[11] D.W. Berreman, Phys. Rev. Lett. 28, 1683 (1972).

[12] Minimization was performed numerically by the conjugate gradient method as in Ref. [2], except that we used the Frank-Oseen [13] rather than the Landau-de Gennes formalism [2] for the LC elastic energy.

[13] C.W. Oseen, Trans. Faraday Soc. 29, 883 (1933); F. C. Frank, Discuss. Faraday Soc. 25, 19 (1958).

[14] D.W. Berreman and T. J. Scheffer, Phys. Rev. Lett. 25, 577 (1970).

[15] We had taken the system to be glass/ITO/PI/ $5 \mathrm{CB}(5 \mu \mathrm{m}) /$ microtexture $/ \mathrm{Si}$ in applying the $4 \times 4 \mathrm{ma}-$ trix method [14]. The refractive indices $\left(n_{\mathrm{ITO}}=1.7\right.$, $\left.n_{\mathrm{PI}}=1.64\right)$ and thickness $\left(d_{\mathrm{ITO}}=30 \mathrm{~nm}, d_{\mathrm{PI}}=70 \mathrm{~nm}\right)$ of the ITO and PI layers are obtained from the manufacturer and verified with optical measurements on thin films of the material on Si and/or glass. The microtexture layer was approximated by a $30 \mathrm{~nm}$ thick homogeneous dielectric whose refractive index was determined by separate experiments [16]. Dividing $I_{A=90^{\circ}}$ by $I_{A=0^{\circ}}$ minimized, if not eliminated, spurious effects due to the spectral response of the measurement system and variations in the light source.

[16] Since the period of the grooves in the microtexture is suboptical, they will not cause any diffraction order and can be treated as an effective homogeneous medium [17]. To determine the refractive index of the microtexture layer as such, we measured the reflection spectrum of empty cells - constructed with the patterned substrates and PI/ITO/glass - from both areas with and without (but nearby) the pattern. The measurements were then repeated with the cells filled with glycerol $(n=$ 1.49). We then model fit the measured spectra by treating the LC cell as glass/ITO/PI/(air or glycerol)/ microtexture/Si with the refractive index of the microtexture layer taken as the only fitting parameter [15]. Data from both kinds of cells yield a consistent value of 1.475 for the refractive index of the microtexture layer. The $\lambda=0.8$ and $0.4 \mu \mathrm{m}$ microtextures had been treated alike in this context since they give rise to the same optical spectrum in Fig. 3.

[17] D. C. Flanders, Appl. Phys. Lett. 42, 492 (1983).

[18] E. Guyon, P. Pieranski, and M. Boix, Lett. Appl. Eng. Sci. 1, 19 (1973).

[19] L. A. Goodman, J. T. McGinn, C. H. Anderson, and F. Digeronimo, IEEE Trans. Electron Devices 24, 795 (1977).

[20] J. D. Bunning, T. E. Faber, and P. L. Sherrell, J. Phys. (Paris) 42, 1175 (1981)

[21] H. Yokoyama and H. A. van Sprang, J. Appl. Phys. 57, 4520 (1985).

[22] L. M. Blinov and V. G. Chigrinov, Electrooptic Effects in Liquid Crystal Materials (Springer-Verlag, New York, 1994).

[23] A. Rapini and M. Papoular, J. Phys. (Paris), Colloq. 30, C4-54 (1969). 